\title{
Evaluación de la postura crítica de futuros profesores de Educación Primaria como componente de la cultura estadística
}

\section{Primary education prospective teachers' critical attitude evaluation as a component of statistical literacy}

\author{
Elena Molina Portillo \\ Javier Contreras García \\ Audy Salcedo ${ }^{3}$ \\ José Miguel Contreras García ${ }^{4}$
}

\begin{abstract}
Resumen: Durante las últimas décadas, la investigación en educación estadística ha incidido en la necesidad de fomentar el desarrollo de la cultura estadística y en especial, de uno de los elementos fundamentales de ella, la actitud crítica hacia la información basada en datos. Debido a que de los conocimientos y destrezas actuales de los futuros profesores de Educación Primaria para interpretar y argumentar críticamente la información estadística dependerá en el futuro su práctica profesional, vemos necesaria una evaluación que indique si es pertinente o no un refuerzo educativo en su formación actual. En este trabajo, se evalúa la actitud crítica de 653 futuros profesores de Educación Primaria, a la hora de identificar la fuente de procedencia de los datos y la fiabilidad de la información presente en gráficos simples, pero que presentan
\end{abstract}

Fecha de recepción: 28 de abril de 2020. Fecha de aceptación: 20 de octubre de 2020.

1 Departamento de didáctica de la Matemática, Facultad de Ciencias de la Educación, Universidad de Granada, España, elemo@ugr.es, orcid.org/0000-0002-9955-3080.

2 Departamento de didáctica de las Ciencias Sociales, Facultad de Ciencias de la Educación y del Deporte de Melilla, Universidad de Granada, España, jcontreras@ugr.es, orcid.org/0000-0003-4064-580X.

3 Departamento de Estadística e Informática Aplicada a la Educación. Facultad de Humanidades y Educación. Universidad Central de Venezuela, audy.salcedo@ucv.ve, orcid.org/0000-0002-9783-8509.

4 Departamento de didáctica de la Matemática, Facultad de Ciencias de la Educación, Universidad de Granada, España, jmcontreras@ugr.es, orcid.org/0000-0001-6821-0563. 
algún tipo de sesgo en su edición. Se ha realizado un análisis descriptivo e inferencial que pone de manifiesto la dificultad a la hora de interpretar críticamente la información presente en ellos, especialmente en los gráficos de sectores, áreas y pictogramas.

Palabras clave: Cultura estadística, futuros profesores, educación primaria, actitud crítica.

\begin{abstract}
For recent decades, research in statistical education has emphasized the need to promote the development of statistical literacy and especially, one of its key elements, the critical attitude towards data-based information. Because the teachers' future professional practice will depend on current knowledge and skills to critically interpret and argue statistical information, an evaluation is required to indicate whether an educational reinforcement of their current training is relevant. In this work, critical attitude of a sample of 653 prospective teachers of primary education when identifying the data source and the reliability of the information present in simple graphs is evaluated, but that present some type of bias in their edition. A descriptive and inferential analysis has been carried out that reveals the difficulty when critical interpretation of their information, especially in the pie charts, area graphs and pictograms.
\end{abstract}

Keywords: Statistical literacy, prospective teachers, primary education, critical attitude.

\title{
1. INTRODUCCIÓN
}

La estadística es parte de la educación general cuyo dominio es deseable en los ciudadanos adultos, quienes precisan adquirir la capacidad de interpretar elementos estadísticos que con frecuencia se les presentan, tales como los que aparecen en los medios de comunicación. Según Lipič y Ovsenik (2020) los ciudadanos con destrezas estadísticas participan de forma más activa y completa en su entorno, tanto social, laboral como personal, siendo capaces de responder a los cambios de forma más eficaz. Además, su estudio ayuda al desarrollo individual, fomentando un razonamiento crítico basado en la valoración de la evidencia. Es por ello que, en muchos países, existe una tendencia conducente a la inclusión de la estadística, y el refuerzo de la cultura estadística de los estudiantes, en los 
currículos oficiales (Ben-Zvi y Makar, 2016). La escuela juega un papel fundamental en el desarrollo de la competencia estadística de los estudiantes, quienes a su vez entienden por qué, y cómo, las estadísticas son útiles para percibir e interpretar el mundo y su complejidad (Frankenstein, 1998). Sin embargo, muchos profesores no se consideran bien preparados para enseñar estadística, ni para enfrentar las dificultades de sus estudiantes (Arteaga, Batanero, Cañadas y Contreras, 2011). Es por ello que, a menudo, los profesores de matemáticas ven la estadística como un capítulo marginal en el plan de estudios de matemáticas y, por tanto, minimizan o ignoran su enseñanza (Ben-Zvi y Makar, 2016).

La noción de cultura estadística surge con la idea de reconocer la necesidad de interpretar, evaluar críticamente y comunicar la información estadística, así como el desarrollo de dichas destrezas desde los primeros cursos de educación básica (Ben-Zvi y Garfield, 2004). Por tanto, la cultura estadística se presenta como un resultado esperado de la escolarización (Garfield y Ben-Zvi, 2007). La definición de qué es ser estadísticamente culto o alfabeto es demasiado amplia (Rumsey, 2002). Sin embargo, a menudo se resalta la importancia de la actitud crítica como componente de la cultura estadística. De este modo Wallman (1993), precursora del término, la define como la habilidad de entender y evaluar críticamente los resultados estadísticos que inundan nuestra vida diaria, unida a la habilidad de apreciar las contribuciones que el razonamiento estadístico puede hacer en público y en privado a las decisiones personales y profesionales. Por su parte Schield (1999) basa su definición en la capacidad de pensar críticamente sobre la estadística como evidencia de los argumentos. Destaca en la literatura la definición de Gal (2002, p.2-3), que distingue dos componentes básicos interrelacionados: a) "capacidad para interpretar y evaluar críticamente la información estadística, los argumentos apoyados en datos o los fenómenos estocásticos que las personas pueden encontrar en diversos contextos, incluyendo los medios de comunicación, pero no limitándose a ellos, y b) capacidad para discutir o comunicar sus opiniones respecto a tales informaciones estadísticas cuando sea relevante".

Uno de los elementos fundamentales para ser estadísticamente culto, es ser capaz de cuestionarse el significado de los elementos estadísticos, evaluándolos críticamente. En este aspecto, Gal (2002) propone fomentar las bases de conocimiento que apoyan la cultura estadística mediante el desarrollo de una postura crítica, creencias de apoyo y actitudes positivas sobre el dominio de la estadística y de uno mismo. Sin embargo, la investigación en educación estadística que aborda el tema de gráficos estadísticos ha centrado su atención, principalmente, en la lectura e interpretación de estos (Rodríguez y Sandoval 
(2012); Carvalho, Campos y Monteiro, 2011; Molina-Portillo, Contreras, Godino, y Díaz-Levicoy, 2017) y, ocasionalmente, en su construcción (Arteaga, Batanero, Contreras y Cañadas, 2015). En este ámbito de estudio, no hay investigaciones que aborden, de forma detallada, el análisis de la fuente de procedencia de los datos y cómo esta influye en la fiabilidad de la información presentada. En la actualidad, la relevancia de esta característica queda reflejada, por ejemplo, en el modelo de estadísticas cívicas (Ridgway, Nicholson y Gal, 2018; ProCivicStat Partners 2018), donde el conocimiento de la producción de datos oficiales es uno de sus componentes. En la misma línea, el valor de los buenos datos dentro de contextos sociales más amplios que las estadísticas, se pone de relieve en la noción de "necesidad de saber" promovida por Ograjensek y Gal (2016). En este aspecto, cabe cuestionarse ¿cómo son los contextos en los que las estadísticas del mundo real son elegidas por los docentes y llevadas al aula? (Gal, 2019). Entre las respuestas aportadas en la literatura, destacamos la realiza por Watson (1997), que señala que los medios de comunicación proporcionan ejemplos (titulares, datos, gráficos o tablas) que podrían servir para relacionar la estadística y la probabilidad con los eventos cotidianos.

Con base en lo expuesto, y dado que los profesores son, en última instancia, los responsables de la transferencia del conocimiento estadístico fundamental, este trabajo evalúa la postura crítica de 653 futuros profesores de Educación Primaria al analizar la fuente de procedencia y la fiabilidad de los datos representados mediante gráficos estadísticos básicos, extraídos de diferentes medios de comunicación.

\section{ACTITUD CRÍTICA EN LA CULTURA ESTADÍSTICA}

En la actual sociedad de la información se tiene la tendencia de asumir como cierta y exacta toda aquella información validada por elementos estadísticos (Hofstadter, 2008). En consecuencia, como incide Bauman (2005), el ciudadano frecuentemente es víctima del abuso de la estadística, debido a que se acepta su uso para explicar cualquier fundamentación, aprovechando la inalterable objetividad de los datos. Por tanto, es fundamental una cultura estadística que permita sacar conclusiones más allá de la interpretación del contenido representado y validado por ellos. Los receptores de la información basada en datos estadísticos deben mostrar interés por la validez de los mensajes, su naturaleza y la credibilidad de las conclusiones que se presentan, así como ser capaces de reflexionar sobre otras posibles interpretaciones (Garfield y Gal, 1999). 
Watson (1997) describió tres niveles de cultura estadística con una sofisticación creciente. El primer nivel conlleva una comprensión básica de la terminología probabilística y estadística; el segundo, una comprensión del lenguaje y conceptos estadísticos cuando están integrados en el contexto de una discusión social más amplia; y, el más sofisticado, implica una actitud de cuestionamiento que uno puede asumir al aplicar conceptos para contradecir afirmaciones hechas sin una base estadística adecuada. Es decir, para la autora, el mayor nivel de cultura estadística radica en una actitud crítica. En una posterior revisión de los niveles de cultura estadística descritos, Watson y Callingham (2003) establecen dos niveles de conocimiento de la estadística: el nivel crítico, que constituye un compromiso cuestionador que implica el uso apropiado de la terminología, la interpretación cualitativa del azar y la apreciación de la variación; y el nivel crítico matemático, en el que se exige un compromiso cuestionador con el contexto, particularmente en contextos de medios o de azar, mostrando aprecio por la necesidad de incertidumbre al hacer predicciones e interpretando aspectos sutiles del lenguaje. Ambos niveles se describen como necesarios para que los estudiantes se conviertan en consumidores inteligentes que puedan tomar decisiones críticas e informadas.

La importancia de la actitud crítica no solo radica en el cuestionamiento. Walshaw (2007) indica que la crítica puede permitir a una persona leer el mundo, descubrir las estructuras ocultas y el discurso que constituyen, así como dar forma a las verdades regulativas de nuestra vida cotidiana. Según Aizikovitsh-Udi et al. (2016), las habilidades del pensamiento crítico se basan en la autorregulación de los procesos de pensamiento, construcción de significados y detección de patrones en estructuras supuestamente desorganizadas. Los autores señalan que el pensamiento crítico tiende a ser complejo y que a menudo termina en soluciones múltiples, en lugar de tener una única solución. En este sentido, Weiland (2017) señala que la cultura estadística no consiste solo en consumir mensajes estadísticos de una manera críticamente evaluativa basándose en conceptos y prácticas de la estadística, sino utilizar la estadística como lente para realizar una nueva visión del mundo. Dicho autor, en su marco de cultura estadística crítica, propone dos niveles: el nivel más bajo, al que denomina nivel de cultura estadística, hace referencia a que el receptor de los datos debe dar sentido y criticar los argumentos basados en datos estadísticos encontrados en diversos contextos. En dicho nivel, el destinatario debe, además, evaluar la fuente, la recopilación y la generación de informes que contengan información estadística. El nivel más alto, al que denomina cultura estadística crítica, hace referencia a que el receptor de los datos debe 
dar sentido al lenguaje y a los sistemas de símbolos estadísticos; además, debe cuestionar la información estadística y los argumentos basados en datos que se encuentran en diversos contextos. En este nivel se debe identificar e interrogar estructuras sociales que dan forma y están reforzadas por argumentos basados en datos; se debe comprender la ubicación social, la subjetividad, el contexto político y tener una visión socio-histórica y política del conocimiento de uno mismo, así como una comprensión de cómo influye esta en la interpretación de la información. Por último, el destinatario debe evaluar la fuente, la recopilación y el informe basado en información estadística y cómo se ven influenciados por la posición social del autor, y la lente sociopolítica e histórica. Todo ello le permitirá tomar conciencia de las estructuras sistémicas en juego de la sociedad.

En el caso de los futuros profesores de Educación Primaria, estos necesitan adquirir no solo conocimientos estadísticos, sino también conocimientos profesionales para la enseñanza de la estadística. De igual forma, se les debe transmitir que un ambiente de aprendizaje efectivo y positivo, puede desarrollar, en los estudiantes, una comprensión profunda y significativa de la estadística, así como la capacidad de pensar y razonar críticamente (Pfannkuch y Ben-Zvi, 2011). Es por ello, que como refiere Engel (2017), se espera que los profesores inculquen en sus estudiantes las capacidades necesarias para que se conviertan en ciudadanos activos que puedan entender las estadísticas de la esfera pública (medios de comunicación, productores de estadísticas oficiales, etc.); y puedan comprobar por su cuenta, a partir de una base de conocimientos sólida que incluya el conocimiento del contexto y las matemáticas básicas; que estén familiarizados con (al menos) estadísticas elementales, las herramientas gráficas y numéricas apropiadas para la representación de datos; y que tengan una actitud crítica y una disposición a comprometerse con la evidencia. Sin embargo, como indican Molina-Portillo et al., (2017), la interpretación crítica de los gráficos estadísticos extraídos de medios de comunicación, supone una gran dificultad para los futuros profesores de Educación Primaria.

\section{METODOLOGÍA DE EVALUACIÓN Y MUESTRA}

La presente investigación toma de referencia la definición y modelo de cultura estadística propuesta por Gal (2002). Para el autor, ser estadísticamente culto no solo depende de los conocimientos que se posean de dicha materia. De este modo, establece que una de las capacidades necesarias para interpretar y 
evaluar la información estadística es una actitud o postura crítica hacia los datos. Esta componente del modelo considerado incide en la capacidad de interpretar y evaluar críticamente la información estadística y los argumentos basados en datos que aparecen en diversos canales, así como en la capacidad para discutir las opiniones con respecto a dicha información estadística. Por tanto, el cuestionamiento crítico de la información estadística permitirá, no solo realizar una interpretación correcta del contenido estadístico sino también, entre otras cosas, conocer la fiabilidad de la información fundamentada en datos.

La población de interés son los futuros profesores de Educación Primaria. En consecuencia, se considera a los alumnos matriculados durante el curso 20182019 en el tercer curso del Grado de Maestro en Educación Primaria de la Universidad de Granada. Esto supuso un total de 653 participantes de los cuales $38,9 \%$ eran chicos y $61,1 \%$ chicas. Dado que la investigación se basa en el contenido estadístico de gráficos simples, los futuros profesores deben tener conocimientos suficientes de estos. Este requisito se presupone como cierto debido a que dichos contenidos forman parte del currículo de matemáticas de Educación Primaria, Secundaria y Bachillerato que cursaron (MEC, 2006a, 2006b, 2007, 2014a; 2014b). Además, dichos estudiantes han cursado durante el Grado de Educación Primaria dos asignaturas previas relacionadas con la matemática y su didáctica, en las cuales han estudiado los gráficos estadísticos elementales, aunque el tiempo dedicado a ello se estima que ha sido de una o dos semanas por curso.

Como instrumento de recogida de información para evaluar la cultura estadística de los futuros profesores de Educación Primaria se ha diseñado y validado un cuestionario específico (Contreras et al., 2017). Dicha herramienta está constituida por un conjunto de cinco noticias que utilizan gráficos estadísticos simples, creados de forma sesgada o incorrecta por algún medio de comunicación tales como la prensa o la televisión. Para cada noticia se pretende evaluar ciertos aspectos de la cultura estadística relacionados con la interpretación de los gráficos estadísticos elementales: un diagrama de barras, de sectores, de líneas, de áreas y un pictograma. Las tareas a realizar ponen en juego conocimientos estadísticos básicos sobre dichos gráficos, evalúan la capacidad de interpretación estadística, examinan el conocimiento del contexto y valoran la actitud crítica en la interpretación.

El cuestionario se administró al inicio de la clase agradeciendo la participación en el estudio, sin aportar orientaciones previas para su resolución a los futuros profesores e indicando que, para la realización del cuestionario completo, disponían de dos horas. En concreto, focalizamos el presente estudio sobre el ítem que 
se centra en estudiar la postura crítica que se adopta frente a la procedencia de los datos utilizados en los gráficos y, con base en los cuales, se interpretan las noticias presentadas. En este sentido, entre las cuestiones planteadas en el cuestionario para cada una de las noticias, se les pidió a los futuros profesores de Educación Primaria la realización de la siguiente tarea: "Indica cuál es la fuente de procedencia de los datos y si consideras que la información es fiable". Como se ha mencionado, la elección de este tipo de gráficos no es casual, ya que estos forman parte de los gráficos que se recomienda enseñar en la mayoría de currículos de Educación Primaria, y entre ellos el currículo español por el que se ha regido la Educación Obligatoria de los participantes en el estudio (MEC 2014a; 2014b). Los gráficos estadísticos utilizados se presentan a continuación:

1. Gráfico de dos barras adosadas, informa de una serie temporal de dos variables estadísticas (número de sociedades mercantiles creadas y disueltas en España) entre los años 2008 y 2012 como se presenta en la figura 1. En dicho gráfico se ha omitido el eje de ordenadas, y la escala con la que se representan sendas variables que tienen distintas proporciones.

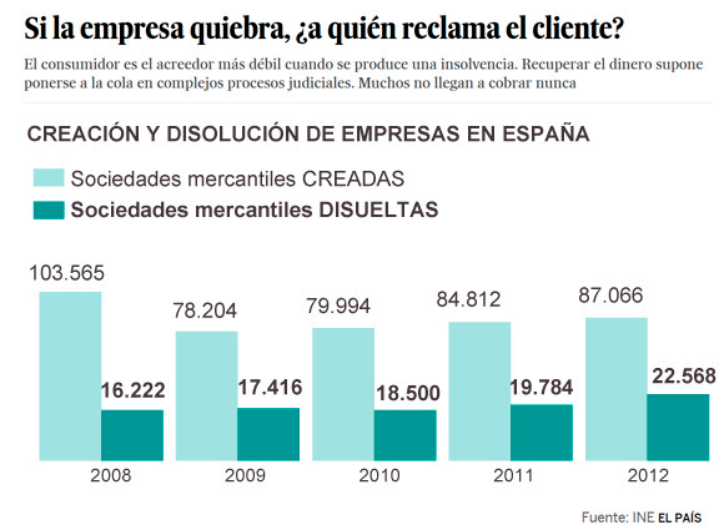

Figura 1. Gráfico de barras adosadas. Noticia extraída del diario elpais.com

El gráfico ha sido publicado por el diario El País y la fuente de procedencia de los datos es el Instituto Nacional de Estadística (INE), organismo oficial que cuenta con medios para que la información sea fiable, en el sentido de que se puede confiar en que los datos están bien recogidos y responden a la realidad. 
2. Gráfico de sectores, representa las votaciones realizadas por un político durante su mandato, figura 2. En este diagrama, el título no refleja bien el contenido, la suma de los porcentajes es $115 \%$ y las áreas de los sectores no guardan la proporción expresada en los porcentajes.

\section{LA SORPRENDENTE MANERA DE PERDER UN DEBATE POLITICO - ESTRATEGIAS ELECTORALES}

October 19, $2013 \cdot$ by vlamel $\cdot$ in Elecciones 2013, Politica - Leave a comment

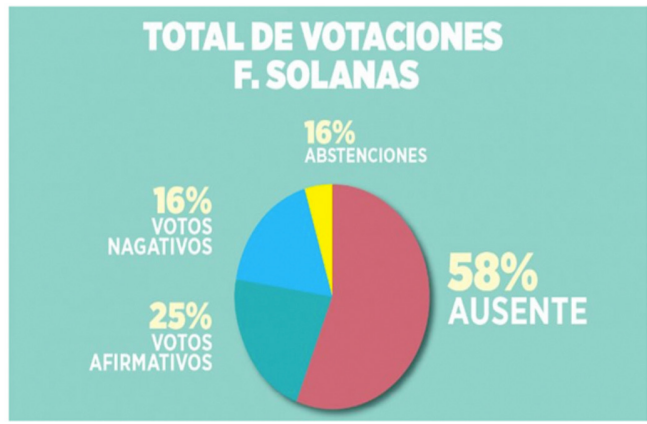

Figura 2. Gráfico de sectores. Noticia extraída de la web limmeridge.wordpress.com

Es un gráfico presentado durante un debate por el propio político. En dicha representación no aparece de forma explícita la fuente de procedencia de los datos. Sin embargo, el conocimiento del contexto indica que los datos deberían ser fiables, ya que se suponen recogidos de los que aportan las diferentes cámaras en pro de transparencia. El problema es que los errores que muestra el gráfico, unido a que en ninguna parte se muestran los datos originales, provoca desconfianza sobre su fiabilidad.

3. Gráfico de líneas, muestra el número total de hipotecas constituidas en España desde 2007 hasta 2014, figura 3. El eje de ordenadas no ha sido incluido en el gráfico y, por tanto, no se indica la escala que se usa para representar las cantidades. Como consecuencia, la representación no respeta la proporcionalidad entre los distintos valores de la variable ni las alturas a las cuales se dibujan los puntos correspondientes. 


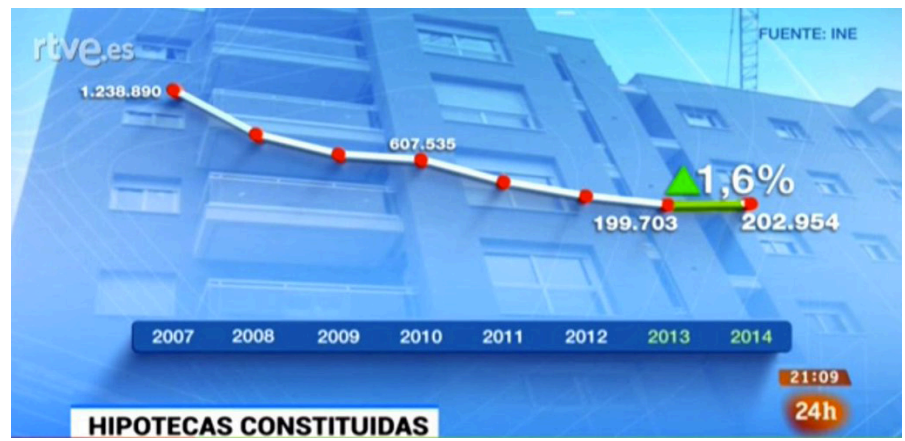

Figura 3. Gráfico de líneas. Gráfica extraída del Canal 24 horas.

El gráfico elaborado por Televisión Española a partir de los datos publicados por el INE, los cuales deben ser fiables, por lo anteriormente expuesto.

4. Gráfico de áreas, alude al coste de los salarios de los empleados públicos desde 1985 a 2011. La figura 4, expone el crecimiento del gasto en remuneraciones durante el periodo 1985-2008, principalmente entre 1985-2000, y el estancamiento o reducción mínima en los años 2008-2011 respecto al aumento experimentado previamente. Para ello, se utilizan intervalos de igual amplitud referidos a distintos periodos de tiempo: 15 años en el primer intervalo; cinco en el segundo; tres en el tercero y periodos de un año en el resto de años representados. Con ello se logra un efecto de incremento brusco de las remuneraciones del sector público en los primeros intervalos que no coincide con el incremento real, el cual ha sido más paulatino.

\section{Los funcionarios se cansan de cargar con los recortes}

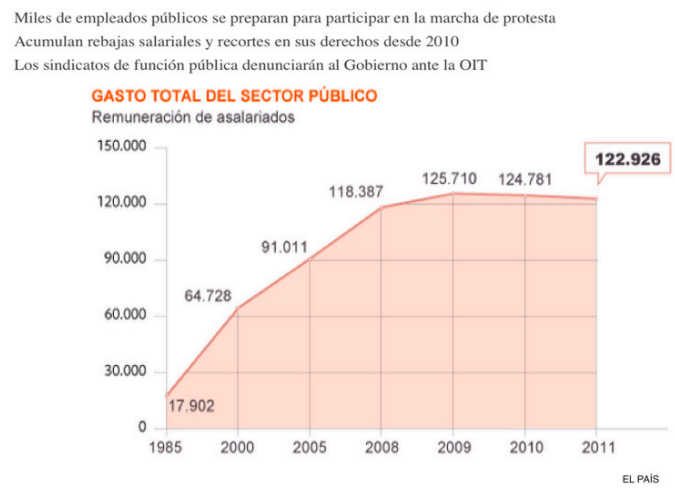

Figura 4. Gráfico de áreas. Noticia extraída del diario elpais.com 
El gráfico ha sido elaborado por el diario El País a partir de los datos publicados por el Ministerio de Empleo y Seguridad Social. Es de destacar que la fuente de procedencia de los datos no aparece en el gráfico, sino únicamente en el cuerpo de la noticia, y en su lugar se indica el nombre del diario en vez de la fuente, lo que puede provocar un sesgo de razonamiento. Los datos deben de ser fiables, ya que la fuente de la información es un organismo oficial que cuenta con medios para su recolección. Sin embargo, dado que no se le facilita la noticia completa, no se espera esta conclusión, sino únicamente se requiere identificar que la fuente no es el medio de comunicación.

5. Pictograma, que representa las ventas de vinos españoles en siete países durante tres años consecutivos (2006, 2007 y 2008). En el gráfico, mostrado en la figura 5, se obvia el eje de ordenadas, lo cual dificulta su interpretación, mientras que la imagen en perspectiva de las botellas, así como el orden en que están colocadas, complica la percepción de la importancia de las cantidades representadas.

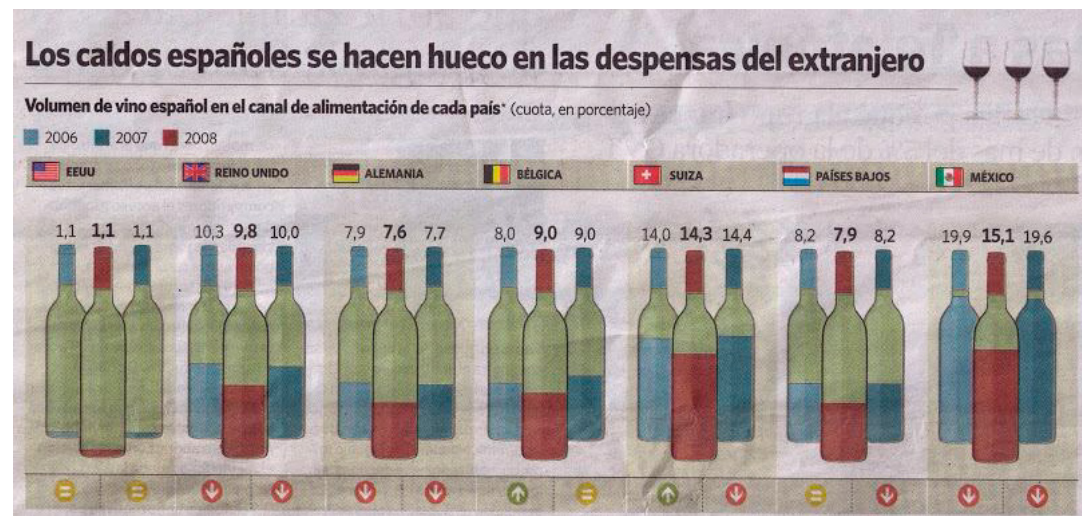

Figura 5. Pictograma. Noticia extraída del diario El Economista.

En este último gráfico no se indica la procedencia de los datos, por lo que, aunque los datos representados sean ciertos, el futuro profesor debe hacer referencia a ello y cuestionarse la fiabilidad de estos.

En cuanto al análisis, se ha realizado un estudio descriptivo clasificando los resultados en función del cuestionamiento o postura crítica adoptada hacia la procedencia de los datos, para cada uno de los gráficos presentados. Para 
tal fin se establecieron dos niveles de concreción: I) si se indica cual es la fuente y se realiza una reflexión sobre la fiabilidad de los datos mostrando una adecuada postura crítica (respuesta correcta, notado por 2); II) si hay un inadecuado cuestionamiento sobre la fuente de procedencia de la información representada (respuesta incorrecta, notado por 0). Destacar en este caso que la fiabilidad atribuida a una fuente de información incorrecta será igualmente inadecuada.

Con el propósito de estudiar los errores que cometen los estudiantes cuando se identifica el origen de los datos y la fiabilidad de estos, se han construido cuatro categorías:

- 0.1 Se identifica como fuente de procedencia de los datos al medio de comunicación que publica la noticia. Para el análisis, esta categoría se ha dividido en tres subcategorías en función de si se considera fiable la información (subcategoría notada por 0.1a), si no se considera fiable (0.1b) o, por el contrario, no se cuestiona la fiabilidad de la información representada (0.1c). En las primeras dos subcategorías, la credibilidad sobre la fiabilidad de la información está condicionada por creencias y actitudes, positivas o negativas, respectivamente, hacia el medio de comunicación que se presupone fuente de los datos representados.

- 0.2 Se identifica una fuente incorrecta diferente al medio de comunicación. Por tanto, tampoco se identifica la verdadera fuente de procedencia de los datos, indicando como tal otra fuente distinta al medio que presenta la noticia, y siendo incorrecto, igualmente, el razonamiento aportado en cuanto a su fiabilidad, en caso de haber sido analizada.

- 0.3 No se identifica la fuente. En este caso, en la resolución de la tarea se indica que no son capaces de identificar la fuente de procedencia y, por tanto, tampoco son capaces de analizar la fiabilidad de los datos.

- 0.4 No responde a la tarea.

Posteriormente a los análisis descritos, se ha comprobado cuál o cuáles de los gráficos analizados presentan mayor número de respuestas correctas a la hora de identificar la fuente de información y constatar la fiabilidad de los datos. Para ello, asignando puntuación a los niveles de concreción descritos (respuesta correcta- 2 puntos- vs respuesta incorrecta -0 puntos), se ha realizado el cálculo de la puntuación media obtenida en cada gráfico y la dispersión de dichas puntuaciones. De forma análoga, para representar la puntuación total obtenida 
por cada participante, se ha definido la variable "suma" y, mediante el cálculo de sus percentiles $25\left(P_{25}\right)$ y $75\left(P_{75}\right)$, se han determinado los grupos de bajo y alto rendimiento, respectivamente.

Con base en lo anterior, para comprobar si existen diferencias significativas en el número de respuestas correctas entre los estudiantes del grupo de bajo rendimiento y los estudiantes del grupo de alto rendimiento, se ha realizado un contraste de hipótesis de igualdad de medias para muestras independientes $(p<0.05)$. Más concretamente, se ha realizado la prueba $U$ de Mann-Whitney para muestras independientes entre los grupos con puntuaciones inferiores al $P_{25}$ y superior al $P_{75}$. A continuación, para descartar que los resultados obtenidos fuesen consecuencia de la distribución asimétrica de la puntuación de las respuestas para cada gráfico, o dicho de otra manera, para refutar que los resultados obtenidos se deban a ítems demasiado fáciles o difíciles, se han calculado los índices de dificultad y discriminación (Morales, 2009), para la tarea correspondiente a cada uno de los gráficos.

Para completar el análisis se consideró necesario estudiar si existe una correlación, positiva o negativa, entre los resultados obtenidos en la tarea para los distintos gráficos propuestos. Así, y dado que la concreción del tipo de respuesta es cualitativa ordinal (respuesta correcta vs respuesta incorrecta), se ha realizado un análisis de correlaciones para escalas ordinales utilizando el coeficiente no paramétrico Tau-b de Kendall.

Finalmente se analiza si el nivel de concreción de las respuestas depende del gráfico estudiado. Dado que las variables generadas por las respuestas a la tarea para los distintos gráficos están relacionadas (respuestas aportadas por cada participante) y que la muestra no es probabilística, se concluye el estudio con la prueba no paramétrica de Wilcoxon. En este caso, la hipótesis nula será que la puntuación obtenida en la tarea (0 si la respuesta es incorrecta o 2 si es correcta) no depende del tipo de gráfico utilizado, comprobando si existe o no diferencias significativas entre las respuestas de los estudiantes a la tarea para cada uno de los gráficos. 


\section{RESULTADOS}

El análisis de las respuestas aportadas por los futuros profesores de Educación Primaria muestra una escasa postura crítica de los estudiantes, principalmente cuando la información solicitada no se encuentra de forma explícita en el gráfico.

El porcentaje de estudiantes que realiza una postura crítica correcta de las distintas noticias, explicando la fuente de procedencia de los datos y si considera que la información es o no fiable, apenas superó $47 \%$. Este porcentaje global de respuestas correctas se consigue, principalmente, gracias a los resultados obtenidos en el gráfico de barras (74,4\%) y de líneas (70,4\%), como se muestra en la tabla 1. En el caso de los otros gráficos, los porcentajes de respuestas correctas se reducen al 38,3\% en el diagrama de sectores, al 34,9\% en el pictograma y al 21,3\% en el gráfico de áreas.

Tabla 1. Frecuencia y porcentaje según tipo de respuesta a la tarea para cada gráfico

\begin{tabular}{llll}
\hline \multicolumn{1}{c}{ Tipo de gráfico } & & Frecuencia (N) & $\begin{array}{c}\text { Porcentaje } \\
\text { (\%) }\end{array}$ \\
\hline \multirow{2}{*}{ Gráfico barras } & Correcto & 486 & 74,4 \\
& Incorrecto & 167 & 25,6 \\
\hline \multirow{2}{*}{ Gráfico de sectores } & Correcto & 250 & 38,3 \\
& Incorrecto & 403 & 61,7 \\
\hline \multirow{2}{*}{ Gráfico de líneas } & Correcto & 460 & 70,4 \\
& Incorrecto & 193 & 29,6 \\
\hline \multirow{2}{*}{ Gráfico de áreas } & Correcto & 139 & 21,3 \\
& Incorrecto & 514 & 78,7 \\
\hline \multirow{2}{*}{ Pictograma } & Correcto & 228 & 34,9 \\
& Incorrecto & 425 & 65,1 \\
\hline
\end{tabular}

A continuación, se muestran los resultados obtenidos al estudiar los errores que cometen los estudiantes al identificar al proveedor de los datos y su fiabilidad (tabla 2). Mediante esta representación de resultados se diferencia claramente la clasificación de errores obtenida para los tres gráficos con más alto porcentaje de respuestas incorrectas: el gráfico de sectores, el pictograma 
y el gráfico de áreas. Las respuestas incorrectas en el diagrama de sectores $(61,7 \%)$ se deben, principalmente, a no identificar la fuente a partir de la información suministrada. En este gráfico destaca que los participantes indicaron no ser capaces de identificar la fuente ni estudiar la fiabilidad de los datos (20,9\% de los errores cometidos respecto al total de respuestas, categoría 0.3 ), siendo aún mayor el porcentaje de estudiantes que atribuye su procedencia y fiabilidad a otra fuente de información (34,6\% de los errores, categoría 0.2). En este último error constatado para dicho gráfico, la mayoría de las respuestas hacen referencia a identificar el término que aparece sobre la gráfica "leave a comment" ("deje un comentario") como fuente de los datos. En el estudio del tipo de error cometido en la tarea para el pictograma, con 65,1\% de respuestas incorrectas, se observa un porcentaje similar para las categorías correspondientes a identificar otra fuente incorrecta (0.2) e identificar como fuente el medio de comunicación sin analizar la fiabilidad (0.1c), 20,2\% y 19,4\% respectivamente. Por último, en el gráfico con mayor porcentaje de errores, el de áreas (78,7\%), destaca el fallo de identificar como fuente de procedencia de los datos al medio de comunicación, considerando fiable la información aportada (42,0\%).

En los gráficos con menor porcentaje de errores, los de barras y de líneas, predomina la primera categoría del análisis por tipo de error. En concreto, para ambos gráficos se identifica al medio de comunicación como la fuente de procedencia de los datos considerados, $18,2 \%$ y $24,1 \%$ respectivamente. Sin embargo, para el gráfico de barras predomina la subcategoría en la que no se consideran fiables los datos (8,4\% de los errores) mientras que para el gráfico de líneas destacan aquellas respuestas en las que no se analiza ni valora la fiabilidad de los datos (15,3\%). Por tanto, estos futuros profesores no hacen una lectura gráfica correcta de la información presente en el gráfico ya que en ambos se indica que la fuente de procedencia de los datos es el INE. 
Tabla 2. Análisis de categorías del tipo de respuestas incorrectas a la tarea para cada gráfico. Número de casos y porcentaje (\%) respecto del total de respuestas

\begin{tabular}{ccrrrr}
\hline Categoría & Gr. barras & Gr. sectores & Gr. líneas & Gr. áreas & Pictograma \\
\hline $0.1 \mathrm{a}$ & $34(5,2)$ & $30(4,6)$ & $48(7,4)$ & $274(42,0)$ & $59(9,0)$ \\
$0.1 \mathrm{~b}$ & $55(8,4)$ & -- & $9(1,4)$ & -- & $36(5,5)$ \\
$0.1 \mathrm{c}$ & $30(4,6)$ & -- & $100(15,3)$ & -- & $127(19,4)$ \\
0.2 & $28(4,3)$ & $226(34,6)$ & $7(1,1)$ & $113(17,3)$ & $132(20,2)$ \\
0.3 & $20(3,1)$ & $136(20,9)$ & $5(0,8)$ & $79(12,1)$ & $56(8,6)$ \\
0.4 & & $11(1,7)$ & $24(3,7)$ & $48(7,4)$ & $15(2,3)$ \\
\hline
\end{tabular}

Nota. La descripción detallada de categorías puede verse en la metodología.

En relación al cálculo de la puntuación media de la tarea para cada uno de los gráficos y su dispersión, y en concordancia con los resultados del análisis de frecuencias, la mayor puntuación media para la tarea se ha obtenido en el diagrama de barras y de líneas, presentando una variabilidad similar para todos los gráficos estudiados (tabla 3). La puntuación media más baja, indicando también la escasez de respuestas correctas, se obtuvo en la tarea que representa la información mediante el gráfico de áreas. Respecto a la puntuación total, o suma de las puntuaciones de la tarea en cada uno de los gráficos, se obtiene una media de 4,79. Así, estos resultados indican que los futuros profesores no alcanzan la puntuación media de 5 , con 53,6\% de puntuaciones inferiores o iguales a 4 y $82,7 \%$ inferiores o iguales a 6 (sobre un máximo de 10), por lo que podemos considerar los resultados obtenidos como deficientes.

Tabla 3. Análisis del tipo de respuesta a la tarea para cada gráfico y suma de los resultados

\begin{tabular}{lcccc}
\hline & Mínimo & Máximo & Media & Desviación estándar \\
\hline Gr. barras & 0 & 2 & 1,49 & 0,873 \\
Gr. sectores & 0 & 2 & 0,77 & 0,973 \\
Gr. líneas & 0 & 2 & 1,41 & 0,913 \\
Gr. áreas & 0 & 2 & 0,43 & 0,819 \\
Pictograma & 0 & 2 & 0,70 & 0,954 \\
Suma total & 0 & 10 & 4,79 & 2,310 \\
\hline
\end{tabular}


La distribución de la puntuación total tiene un $\mathrm{P}_{25}$ y $\mathrm{P}_{75}$ de 2 y 6 , respectivamente. Así, se considera estudiante de bajo rendimiento a aquellos participantes cuya puntuación total es menor o igual a 2. Por el contrario, se considera estudiante de alto rendimiento a aquellos futuros profesores cuya puntuación total es igual o mayor a 6 . Así, se ha realizado la prueba $U$ de Mann-Whitney para muestras independientes entre los grupos de bajo y alto rendimiento. Los resultados indican que se debe rechazar la hipótesis nula de igualdad de medias en todas las tareas, con un p-valor de 0,000 para todos los gráficos, por tanto, existen diferencias significativas en las respuestas aportadas por los grupos de alto y bajo rendimiento.

Los resultados que se muestran en la tabla 4 , indican que la respuesta resulta fácil para los futuros profesores en el caso del diagrama de barras y de líneas, debido principalmente a que la fuente estaba indicada en la gráfica. No ocurre lo mismo en el caso de la tarea sobre el gráfico de sectores y el pictograma, donde los futuros profesores han de interpretar la fuente de procedencia a través del contenido de la noticia. En el caso de la tarea para el gráfico de áreas resulta aún más complicada (menor índice de dificultad), que se considera debido, principalmente, a que la presencia en la gráfica del nombre del medio de comunicación lleva a confundir a dicho medio con la fuente. En el caso del índice de discriminación, los valores de la tarea para el gráfico de sectores y de líneas son cercanos o iguales a 0,5, indicando una fuerte discriminación entre los grupos de alto y bajo rendimiento. En otras palabras, se refleja en estos resultados que para dichos gráficos responden correctamente a la tarea mayoritariamente los estudiantes del grupo de alto rendimiento mientras que, usualmente, los participantes de bajo rendimiento responden incorrectamente. En el resto de tareas, el índice de discriminación es menor que 0,5 lo que indica que las respuestas correctas no difieren tanto entre el grupo de alto y bajo rendimiento. Dicho de otro modo, en el resto de gráficos la complejidad de la tarea produce respuestas incorrectas en ambos grupos, principalmente en la tarea referida al pictograma.

Tabla 4. Índices de dificultad y discriminación por rendimiento para $P_{25}-P_{75}$.

\begin{tabular}{lcc}
\hline & I. dificultad & I. discriminación \\
\hline Gr. barras & 0,74 & 0,33 \\
Gr. sectores & 0,38 & 0,50 \\
Gr. líneas & 0,70 & 0,45 \\
Gr. áreas & 0,21 & 0,38 \\
Pictograma & 0,35 & 0,27 \\
\hline
\end{tabular}


Como se observa en la tabla 5, en todos los contrastes de hipótesis en los que está involucrado el pictograma se acepta la hipótesis nula de "no existencia de correlación entre ambas variables" con un nivel de confianza del 95\%. Es decir, no existe relación entre las respuestas a la tarea que involucra al pictograma con ninguna de las respuestas a la tarea que involucra a cualquier otro gráfico. Para el resto de gráficos podemos indicar que existe correlación entre las respuestas a la tarea planteada, pero todas menores de 0,4 , por lo que se consideran bajas (o muy bajas, si son menores de 0,2). Las mayores correlaciones las encontramos entre las respuestas a la tarea para el gráfico de líneas con las respuestas a la tarea para el diagrama de barras y con las respuestas a la tarea para el gráfico de áreas, ambas positivas.

Tabla 5. Correlaciones Tau-b de Kendall entre las distintas tareas

\begin{tabular}{lllcccc}
\hline & & Gr. barras & Gr. sectores & Gr. líneas & Gr. áreas & Pictograma \\
\hline \multirow{2}{*}{ Gr. barras } & Coeficiente de correlación & -- & 0,101 & 0,282 & 0,090 & $-0,071$ \\
\cline { 2 - 7 } & p-valor & -- & 0,010 & 0,000 & 0,021 & 0,068 \\
\hline \multirow{2}{*}{ Gr. sectores } & Coeficiente de correlación & -- & -- & 0,096 & 0,106 & $-0,022$ \\
\cline { 2 - 7 } & p-valor & -- & -- & 0,014 & 0,007 & 0,579 \\
\hline \multirow{2}{*}{ Gr. líneas } & Coeficiente de correlación & -- & -- & -- & 0,214 & $-0,054$ \\
\cline { 2 - 7 } & p-valor & -- & -- & -- & 0,000 & 0,171 \\
\hline \multirow{2}{*}{ Gr. áreas } & Coeficiente de correlación & -- & -- & -- & -- & 0,035 \\
\cline { 2 - 7 } & p-valor & -- & -- & -- & -- & 0,371 \\
\hline \multirow{2}{*}{ Pictograma } & Coeficiente de correlación & -- & -- & -- & -- & -- \\
\hline & p-valor & -- & -- & -- & -- & -- \\
\hline
\end{tabular}

La tabla 6 presenta los resultados de la prueba de Wilcoxon para muestras relacionadas. Como se observa, solo en dos de las comparaciones se acepta la hipótesis nula de igualdad de medianas, para un nivel de confianza del 95\%. La hipótesis nula de que la puntuación obtenida en la tarea no depende del tipo de gráfico utilizado, se cumple en el caso de la comparación del diagrama de barras con el gráfico de líneas, y en el caso del diagrama de sectores y el pictograma. Por tanto, en estos casos podemos afirmar que no hay diferencias significativas entre las respuestas de los estudiantes a ambas tareas, coincidiendo estos resultados con los índices de dificultad que presentan ambos pares. 
Tabla 6. Prueba Wilcoxon pruebas emparejadas. P-valor

\begin{tabular}{lccccc}
\hline & Gr. barras & Gr. sectores & Gr. líneas & Gr. áreas & Pictograma \\
\hline Gr. barras & -- & 0,000 & 0,058 & 0,000 & 0,000 \\
Gr. sectores & -- & -- & 0,000 & 0,000 & 0,212 \\
Gr. líneas & -- & -- & -- & 0,000 & 0,000 \\
Gr. áreas & -- & -- & -- & -- & 0,000 \\
& & & -- & -- & -- \\
Pictograma & -- & -- & - &
\end{tabular}

\section{CONCLUSIONES DEL ESTUDIO}

Los resultados de esta investigación muestran una deficiente cultura estadística por parte de los futuros profesores de Educación Primaria, participantes en el estudio, concretamente en la componente de postura crítica según el modelo de Gal (2002). Esto indica que no han desarrollado la capacidad de interpretar y evaluar críticamente la información estadística, principalmente cuando la información solicitada no se encuentra de forma explícita en el gráfico o aparecen elementos distractores. Muestra de ello se observa en los resultados obtenidos en el análisis de errores para el diagrama de sectores, pictograma y gráfico de áreas. Sin embargo, aun cuando dichos elementos aparecen de forma explícita, frecuentemente se considera al medio de comunicación que publica la noticia como fuente de procedencia de los datos. Esto evidencia, no solo una inadecuada postura crítica sino también, en línea con Molina-Portillo et al. (2019), una carencia de conocimientos básicos asociados a una correcta lectura de gráficos estadísticos. En este caso es usual, además de no abordar la credibilidad que tendrían los datos, atribuir su fiabilidad en virtud de las creencias personales hacia dichos medios. Estos resultados, en consonancia con Murray y Gal (2002), indican que la postura crítica queda respaldada por las creencias y actitudes personales, hecho que se confirma incluso cuando hay carencias en los niveles de conocimiento de cultura estadística descritos por Gal (2002).

Las carencias encontradas van más allá del gráfico utilizado ya que la mayoría de los errores dependen de cuestiones ajenas al tipo de gráfico o a los sesgos presentes él. Esto indica que la falta de actitud crítica puede estar 
condicionada por desconocimiento, o mala praxis, de elementos fundamentales de la cultura estadística tales como el conocimiento del contexto o los elementos estructurales de los gráficos estadísticos. Por el contrario, los alumnos que realizan correctamente la tarea habrían alcanzado el nivel "cultura estadística crítica" descrito por Weiland (2017), o el "crítico matemático" descrito por Watson y Callingham (2003), ya que son capaces de identificar correctamente la fuente de procedencia de los datos y realizan una interpretación de la fiabilidad de los mismos en función de ello.

En línea con Molina-Portillo et al. (2017) estos resultados inciden en la necesidad de fomentar la cultura estadística de los futuros profesores de Educación Primaria, mostrando una deficiencia en su formación estadística básica relacionada con el conocimiento del contexto y la postura crítica hacia la información basada en datos. Sería deseable una formación complementaria, o una profundización en la formación actual, sobre la importancia de los aspectos que han sido objeto de este estudio. Como señala Gal (2002), se ha de fomentar la capacidad de interpretar y evaluar críticamente la información estadística por parte de los ciudadanos, y en especial de aquellos encargados en formarlos. De lo contrario, puede que no logren ser competentes en su futura tarea como docentes, especialmente a la hora de formar ciudadanos estadísticamente cultos.

El presente trabajo profundiza en el estudio de una de las componentes de cultura estadística que, aunque definida y puesta en valor, ha sido poco analizada en el ámbito de la investigación en educación estadística. Sus resultados constituyen un aporte incuestionable a los obtenidos en trabajos sobre interpretación y creación de gráficos estadísticos (Arteaga et al., 2015), mediante el estudio del mayor nivel de cultura estadística descrita en los modelos teóricos, aquel que requiere una postura crítica (Gal, 2002; Ridgway et al., 2018; Walshaw, 2007; Watson, 1997; Watson y Callingham, 2003; Weiland, 2017; entre otros). Otro aspecto a tomar en consideración es que el instrumento utilizado genera resultados robustos para la componente de postura crítica analizada. En primer lugar, no existen correlaciones, o son muy bajas, entre las respuestas a la tarea para los distintos gráficos. Además, la tarea presenta distintos grados de complejidad según el gráfico analizado, así como distinto grado de eficiencia en correlación con la puntuación global del cuestionario (discrimina correctamente). Esta característica confirma que a mayor complejidad de la tarea se produce una mejor discriminación entre los participantes que responden correctamente y los que no. Del mismo modo, la variabilidad en el índice de dificultad nos permite enfatizar la importancia de los elementos estructurales del gráfico en la 
adopción de una adecuada postura crítica identificándose mayor número de errores o incorrecciones donde dichos elementos o no son explícitos, o bien se sustituyen por otros elementos distractores (como sucede en el diagrama de sectores o gráfico de áreas).

La continuidad de esta línea de investigación pasaría por establecer estudios complementarios que aborden la temática tratada ampliando su ámbito de estudio, tomando muestras probabilísticas cuyos resultados pudiesen ser extrapolables a la población de estudio, o acotándolo y estudiando características concretas del origen de los errores detectados. En este sentido, investigaciones complementarias en el ámbito internacional podrían establecer comparaciones interesantes en el ámbito de la formación estadística de futuros profesores para promover la cultura estadística en la sociedad.

\section{RECONOCIMIENTOS}

Trabajo realizado en el marco del proyecto B-SEJ-063-UGR18 y del Grupo SEJ-622 (Junta de Andalucía).

\section{REFERENCIAS}

Aizikovitsh-Udi, E., Kuntze, S. y Clarke, D. (2016). Connections between statistical thinking and critical thinking: A case study. En The Teaching and Learning of Statistics (pp. 83-94). Springer, Cham.

Arteaga, P., Batanero, C., Cañadas, G. y Contreras, J. M. (2011). Las tablas y gráficos estadísticos como objetos culturales. Números. Revista de didáctica de las matemáticas, 76, 55-67.

Bauman, Z. (2005). Modernidad y ambivalencia (Vol. 44). Anthropos Editorial.

Ben-Zvi, D. y Garfield, J. (2004). Statistical literacy, reasoning, and thinking: Goals, definitions, and challenges. En The challenge of developing statistical literacy, reasoning and thinking (pp. 3-15). Springer.

Ben-Zvi, D. y Makar, K. (2016). International Perspectives on the Teaching and Learning of Statistics. En The Teaching and Learning of Statistics (pp. 1-10). Springer International Publishing. 
Carvalho, L. M., Campos, T. M. y Monteiro, C. E. (2011). Aspectos visuais e conceituais nas interpretaçōes de gráficos de linhas por estudantes. Boletim de Educação Matemática, 24(40), 679-700.

Contreras, J. M., Molina-Portillo, E., Godino, J. D., y Batanero, C. (2017). Construcción de un cuestionario para evaluar la interpretación crítica de gráficos estadísticos por futuros profesores. En Investigación en Educación Matemática XXI (pp. 207-216). Sociedad Española de Investigación en Educación Matemática, SEIEM.

Engel, J. (2017). Statistical Literacy for Active Citizenship: A Call for Data Science Education. Statistics Education Research Journal, 16(1), 44-49.

Frankenstein, M. (1998). Reading the World with Math: Goals for a Critical Mathematical Literacy Curriculum. En E. Lee, D. Menkart, M. Okazawa-Rey (Eds.) Beyond Heroes and Holidays: A Practical Guide to K-12 Anti-Racist, Multicultural Education and Staff Development, Network of Educators on the Americas.

Gal, I (2002). Adult's statistical literacy. Meanings, components, responsibilities. International Statistical Review, 70(1), 1-25.

Gal, I. (2019). Understanding statistical literacy: About knowledge of contexts and models. En J. M. Contreras, M. M. Gea, M. M. López-Martín y E. Molina-Portillo (Eds.), Actas del Tercer Congreso International Virtual de Educación Estadística.

Garfield, J. y Ben-Zvi, D. (2007). How students learn statistics revisited: A current review of research on teaching and learning statistics. International Statistical Review, 75(3), 372-396.

Garfield, J. B. y Gal, I. (1999). Assessment and statistics education: Current challenges and directions. International statistical review, 67(1), 1-12.

Hofstadter, D. (2008). Metamagical themas: Questing for the essence of mind and pattern. Basic books.

Lipič, N. y Ovsenik, M. (2020). The Effect of Statistical Literacy on Response to Environmental Change. Organizacija, 53(2), 147-163.

MEC (2006a). Real Decreto 1513/2006, de 7 de diciembre, por el que se establecen las enseñanzas mínimas correspondientes a la Educación Primaria. Ministerio de Educación y Ciencia.

MEC (2006b). Real Decreto 1631/2006, de 29 de diciembre, por el que se establecen las enseñanzas mínimas correspondientes a la Educación Secundaria Obligatoria. Ministerio de Educación y Ciencia.

MEC (2007). Real Decreto 1467/2007, de 2 de noviembre, por el que se establece la estructura de bachillerato y se fijan sus enseñanzas mínimas. Ministerio de Educación y Ciencia. 
MEC (2014a). Real Decreto 126/2014, de 28 de febrero, por el que se establece el currículo básico de la Educación Primaria. Ministerio de Educación y Ciencia

MEC (2014b). Real Decreto 1105/2014, de 26 de diciembre, por el que se establece el currículo básico de la Educación Secundaria Obligatoria y del Bachillerato. Ministerio de Educación y Ciencia.

Molina-Portillo, E., Contreras, J. M., Godino, J. D. y Ruz, F. (2019). Statistical literacy in the information society. BEIO, Boletín de Estadística e Investigación Operativa, 35(2), 148-169.

Molina-Portillo, E., Contreras, J. M., Godino, J. D., y Díaz-Levicoy, D. (2017). Interpretación crítica de gráficos estadísticos incorrectos en la sociedad de la comunicación: un desafío para futuros maestros. Enseñanza de las Ciencias, 4787-4792.

Morales, P. (2009). Análisis de ítems en las pruebas objetivas. Universidad Pontificia Comillas.

Murray, S. y Gal, I. (2002). Preparing for diversity in statistics literacy: Institutional and educational implications. In Proceedings of the Sixth International Conference on Teaching of Statistics. IASE. CD ROM.

Pfannkuch, M. y Ben-Zvi, D. (2011). Developing teachers' statistical thinking. En C. Batanero, G. Burrill, y C. Reading (Eds.), Teaching statistics in school mathematics: Challenges for teaching and teacher education. Springer.

Ograjensek, I. y Gal, I. (2016). Enhancing statistics education by including qualitative research. International Statistical Review, 84(2), 165-178. https://doi.org/10.1111/ insr.12158

ProCivicStat Partners. (2018). Engaging civic statistics: A call for action and recommendations. (A product of the ProCivicStat Project). Retrieved March 10, 2020, https:// iase-web.org/islp/pcs/

Ridgway, R., Nicholson, J. y Gal, I. (2018). Understanding statistics about society: A brief framework of knowledge and skills needed to engage with civic statistics. http:// iaseweb.org/icots/10/proceedings/pdfs/ICOTS10_7A1.pdf

Rodríguez, F. y Sandoval, P. (2012). Habilidades de codificación y descodificación de tablas y gráficos estadísticos: un estudio comparativo en profesores y alumnos de pedagogía en Enseñanza Básica. Avaliaçāo, 17(1), 207-235.

Rumsey, D. (2002). Statistical Literacy as a Goal for Introductory Statistics Courses. ASA Proceedings of the Section on Statistical Education.

Schield, M. (1999). Statistical literacy: Thinking critically about statistics. Of Significance, 1(1), 15-20.

Wallman, K. K. (1993). Enhancing statistical literacy: Enriching our society. Journal of the American Statistical Association, 88(421), 1-8. 
Walshaw, M. (2007). Working with Foucault in education. Sense Publishing.

Watson, J. M. (1997). Assessing statistical literacy using the media. En I. Gal y J. B. Garfield

(Eds.), The assessment challenge in statistics education (pp. 107-121). IOS Press and The International Statistical Institute.

Watson, J. y Callingham, R. (2003). Statistical literacy: A complex hierarchical construct. Statistics Education Research Journal, 2(2), 3-46.

Weiland, T. (2017). Problematizing statistical literacy: An intersection of critical and statistical literacies. Educational Studies in Mathematics, 96(1), 33-47.

José Miguel ContreRAS García

Dirección: Departamento de Didáctica de la Matemática, Facultad de Ciencias de Educación Universidad de Granada, Campus de Cartuja, 18071 Granada (España).

Teléfono: $\quad 958242846$ 